\title{
Высокая характеристическая температура лазера на квантовых точках InAs/GaAs/InGaAsP с длиной волны излучения около 1.5 мкм, синтезированного на подложке InP
} \author{
М.B. Максимов ${ }^{1}$, А.Е. Жуков ${ }^{1}$ \\ ${ }^{1}$ Академический университет Российской академии наук, \\ 194021 Санкт-Петербург, Россия \\ ${ }^{2}$ DTU Fotonics Technical University of Denmark, Kgs. Lyngby, \\ DK-2800 Denmark \\ E-mail: fedyazu@mail.ru
}

(C) Ф.И. Зубов ${ }^{1}$, Е.С. Семенова ${ }^{2}$, И.В. Кулькова ${ }^{2}$, К. Yvind ${ }^{2}$, Н.В. Крыжановская ${ }^{1}$,

(Получена 27 марта 2017 г. Принята к печати 5 апреля 2017 г.)

Представлены результаты исследования синтезированных на подложке $\operatorname{InP}(100)$ лазеров с длиной волны излучения около 1.5 мкм, характеризующихся высокой температурной стабильностью. В качестве активной области лазера были использованы самоорганизованные квантовые точки InAs, покрытые тонким слоем GaAs. Волноводным/матричным слоем являлся четверной твердый раствор InGaAsP с шириной запрещенной зоны 1.15 эВ. Высокая характеристическая температура порогового тока $T_{0}=205 \mathrm{~K}$ в температурном диапазоне $20-50^{\circ} \mathrm{C}$ была достигнута в лазерных диодах с гребешковым волноводом. Была обнаружена взаимосвязь между значениями $T_{0}$ и шириной запрещенной зоны волноводных слоев.

DOI: 10.21883/FTP.2017.10.45017.8590

\section{1. Введение}

В последние более чем двадцать лет активно ведутся исследования лазерных диодов на основе самоорганизующихся квантовых точек (КТ) [1]. Наиболее значительные результаты были достигнуты для лазеров с КТ на подложках $\mathrm{GaAs}$ (в особенности для излучающих в спектральном диапазоне вблизи 1.3 мкм [2,3]). Такие лазеры продемонстрировали рекордно низкую пороговую плотность тока [4], близкий к нулю фактор уширения лазерной линии [5], а также температурно-независимые пороговые характеристики [6]. Лазеры с КТ на подложках InP являются перспективными приборами для использования в телекоммуникационных системах с длиной волны генерации около 1.5 мкм (например, $C$-диапазону оптической связи соответствуют длины волн 1530-1565 нм). KT InAs, излучающие в необходимом спектральном диапазоне, могут быть синтезированы как методом молекулярно-пучковой эпитаксии (МПЭ) [7], так и газофазной эпитаксии из металлоорганических соединений (ГФЭ МОС) [8]

Использование стандартных ростовых условий МПЭ и точно ориентированных подложек $\operatorname{InP}(100)$ [9] приводит к формированию так называемых квантовых штрихов (наноостровков, вытянутых вдоль направления $[1 \overline{1} 0])$, тогда как ГФЭ МОС позволяет осаждать на поверхности InP (100) КТ с аспектным отношением в плоскости роста, близким к 1. Для КТ, синтезированных на подложках InP, типично большое неоднородное уширение энергетических состояний, которое в несколько раз превосходит значения, характерные для $\operatorname{In}(\mathrm{Ga}) \mathrm{As}$ KT, синтезированных на подложках GaAs. К настоящему времени был разработан ряд подходов к управлению свойствами InAs/InP KT. Для подавления удлинения KT, выращиваемых методом МПЭ, был предложен синтез на нестандартных (311) [10] или вицинальных подложках [11], рост активной области при пониженных температурах около $480^{\circ} \mathrm{C}[10,12-14]$, а также синтез в атмосфере $\mathrm{As}_{2}$ вместо $\mathrm{As}_{4}[14,15]$. Для улучшения однородности КТ, синтезированных методом ГФЭ МОС, использовались тонкие подслои GaAs [16] или GaP [17], а также низкая ростовая температура $470^{\circ} \mathrm{C}$ [18]. В результате в лазерах с широким полоском с активной областью на основе КТ с уменьшенным неоднородным уширением была достигнута низкая пороговая плотность тока $190 \mathrm{~A} / \mathrm{cm}^{2}$ [12], высокое оптическое усиление около $10 \mathrm{~cm}^{-1}$ на один слой КТ $[13,14]$, мощность лазерного излучения 1.1 Вт в непрерывном режиме [16].

Большое неоднородное уширение InAs/InP KT обычно рассматривается как нежелательное явление, поскольку оно снижает максимальное/насыщенное усиление и приводит к росту плотности порогового тока. В то же время оно позволяет реализовать широкие спектры усиления и лазерной генерации, необходимые для генерации сверхкоротких импульсов в режиме синхронизации мод [19]. Совсем недавно за счет использования преднамеренного внесения неоднородности в процессе синтеза InAs/InP КТ [20] был получен сверхширокий (92 нм) с плоской вершиной спектр лазерной генерации с центральной длиной волны около 1.56 мкм. В свою очередь, как было ранее продемонстрировано для КТ на подложках $\mathrm{GaAs}$, применение лазеров с синхронизацией мод позволяет подвинуться в область частот свыше 200 ГГц [21], недоступную для прямой модуляции вследствие характерного для КТ высокого значения коэффициента компрессии усиления [22]. 
В пространственно-одномодовых лазерах, требуемых для использования в системах оптической связи, пороговая плотность (типично уровня кА/ $\mathrm{cm}^{2}$ ) заметно выше, чем в лазерах с широким полоском, что обусловлено влиянием боковых стенок травления в сочетании с относительно низким усилением, которым характеризуются КТ, оптимизированные для применений в режиме синхронизации мод. Однако одной из основных трудностей, которую необходимо преодолеть, является плохая температурная стабильность. Для 1.5 мкм КТ лазеров, синтезированных на подложке InP, типичное значение характеристической температуры порогового тока $T_{0}$ составляет около $100 \mathrm{~K}$ [23].

В настоящей работе было достигнуто значительное улучшение температурной стабильности лазера на КТ с длиной волны генерации около 1.5 мкм, синтезированного на подложке InP. Использование в качестве волноводного/матричного слоя материала InGaAsP c большой шириной запрещенной зоны в сочетании с покрытием КT InAs слоем GaAs позволило в лазере с полоском шириной 2 мкм достичь характеристических температур порогового тока и внешней дифференциальной эффективности 205 и $235 \mathrm{~K}$ соответственно.

\section{2. Эксперимент}

Лазерная структура была выращена методом ГФЭ MOC низкого давления на подложке $n^{+}-\mathrm{InP}(001)$. Волновод InGaAsP толщиной 450 нм располагался между InP эмиттерными слоями $n$ - и $p$-типа. Ширина запрещенной зоны InGaAsP (Q1.08) была выбрана равной 1.15 эВ. Хотя использование широкозонного волновода и приводит к снижению фактора оптического ограничения (и, как результат, к увеличению пороговой плотности тока при комнатной температуре) по сравнению с лазером с более узкозонным волноводом, это позволяет подавить выброс носителей заряда из активной области, являющийся одной из причин роста порога генерации при увеличении температуры. Пять слоев КТ, сформированных посредством осаждения 1.65 монослоя (MC) InAs и разделенных спейсерами InGaAsP (Q1.08) 60 нм, образовывали активную область, помещенную в центр волновода. Активная область выращивалась при температуре $516^{\circ} \mathrm{C}$, тогда как остальная часть структуры синтезировалась при $610^{\circ} \mathrm{C}$. Чтобы получить нужную длину волны около 1.5 мкм, каждый слой КТ покрывался тонким (1.7 MC) слоем GaAs (см. подробности в работе [24]). Результаты исследования атомно-силовой микроскопии и сканирующей просвечивающей туннельной микроскопии, показали, что поверхностная плотность КТ составляет $\sim 4 \cdot 10^{10} \mathrm{~cm}^{-2}$ в одном слое, а средние размеры КТ $-31.5 \pm 2$ нм диаметр основания и 2.6 нм по высоте. Режимы осаждения позволяли достичь высокой степени неоднородности массива КТ с целью их последующего применения в лазерах с синхронизацией мод: ширина линии фотолюминесценции на половине высоты при комнатной температуре составила около 100 мэВ.

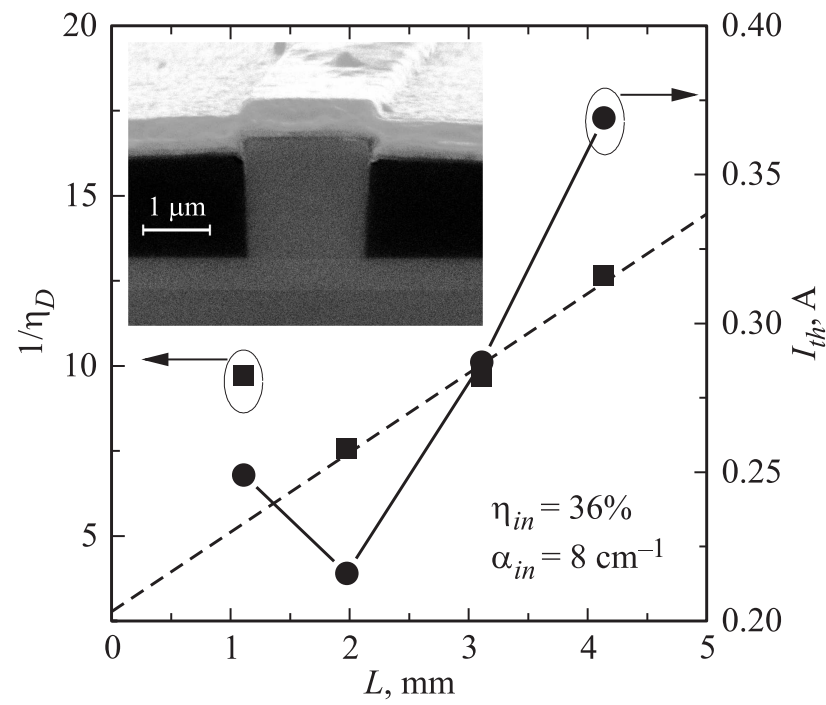

Рис. 1. Обратная внешняя дифференциальная эффективность и пороговый ток в зависимости от длины резонатора при $20^{\circ} \mathrm{C}$. Штриховая линия - линейная аппроксимация. На вставке СЭМ-снимок лазерного гребешка.

Лазеры с гребешковым волноводом были изготовлены из эпитаксиальной структуры с использованием стандартной оптической литографии и реактивного ионного травления. Остаточная толщина верхнего эмиттера была выбрана 40 нм. Для планаризации поверхности использовался бензоциклобутен (Cyclotene 3046). Для формирования $p$-контакта применялась металлизация $\mathrm{Ti} / \mathrm{Pt} / \mathrm{Au}$. После утоньшения подложки для формирования $n$-контакта была осаждена металлизация $\mathrm{Ni} / \mathrm{Ge} / \mathrm{Au}$. На лазерные грани, полученные скалыванием, покрытие не наносилось. На полученной с помощью сканирующего электронного микроскопа (СЭМ) микрофотографии, представленной на вставке к рис. 1, показан лазерный полосок шириной 2 мкм. Были изготовлены лазерные диоды различной длины от 1 до 4 мм. Исследуемые лазеры были напаяны на медные теплоотводы $p$-стороной вверх.

Если не указано иное, то далее мы приводим характеристики, измеренные при импульсной накачке (импульсы 5 мкс, фактор заполнения $0.1 \%$ ). Измерения проводились в диапазоне $20-70^{\circ} \mathrm{C}$. Максимальная температура была ограничена контроллером температуры. Оптическая мощность определялась с использованием Ge-фотодиода Thorlabs большой площади $1 \mathrm{~cm}^{2}$. Спектры электролюминесценции записывались при помощи монохроматора Spectral Products в сочетании с InGaAs-фотодиодом Teledyne Judson.

\section{3. Результаты и обсуждение}

Лазерные диоды имели низкое удельное последовательное сопротивление $3.6 \cdot 10^{-5} \mathrm{OM} \cdot \mathrm{cm}^{2}$ и напряжение открывания $0.98 \mathrm{~B}$. На рис. 1 показана зависимость порогового тока и обратной внешней дифференциальной 


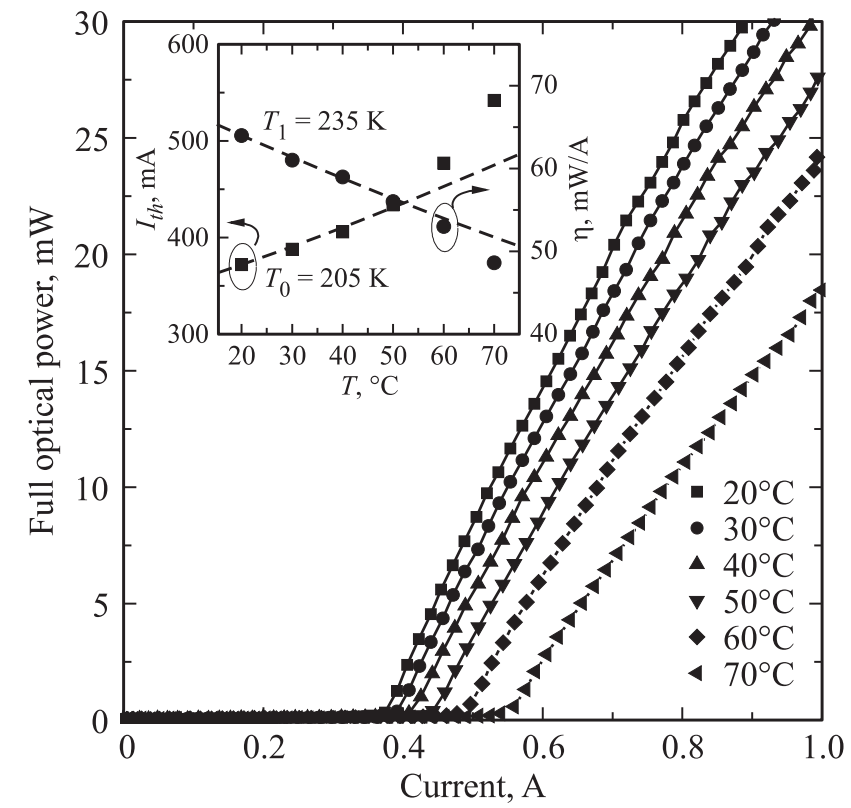

Рис. 2. Ватт-амперные характеристики 4 мм КТ лазера при различных температурах. На вставке - пороговый ток и внешняя эффективность в зависимости от окружающей температуры 4 мм КТ лазера. Штриховые линии - экспоненциальные аппроксимации.

эффективности $\left(1 / \eta_{D}\right)$ от длины резонатора, которая была использована для определения внутренних потерь $\alpha_{\text {in }}=8 \mathrm{~cm}^{-1}$ и внутренней дифференциальной квантовой эффективности $\eta_{\text {in }}=36 \%$. Такие значения типичны для лазеров на KT InAs/InP спектрального диапазона 1.5 мкм [23]. Для корректного определения $\alpha_{\text {in }}$ и $\eta_{\text {in ис- }}$ пользовались данные для лазеров с большими длинами ( $\geq 2 \mathrm{MM})$. Пороговый ток уменьшался от 0.37 до $0.22 \mathrm{~A}$ по мере изменения длины резонатора от 4 до 2 мм, а затем вырастал до 0.25 А в образце длиной 1 мм.

На рис. 2 показаны ватт-амперные характеристики, измеренные при различных температурах в диапазоне $20-70^{\circ} \mathrm{C}$ для лазера длиной 4 мм. Линейная зависимость выходной мощности от тока накачки сохранялась по крайней мере до 20 мВт во всем исследованном диапазоне температур. В непрерывном режиме генерации при $20^{\circ} \mathrm{C}$ оптическая мощность достигала около $10 \mathrm{MBT}$ и была лимитирована тепловым загибом.

Несмотря на высокий пороговый ток (0.37 А при $\left.20^{\circ} \mathrm{C}\right)$, лазер демонстрировал высокую температурную стабильность характеристик. Зависимости порогового тока $\left(I_{\mathrm{th}}\right)$ и внешней дифференциальной эффективности $(\eta)$ от температуры представлены на вставке к рис. 2. Посредством аппроксимации экспоненциальными функциями $I_{\text {th }}=I_{0} \exp \left(T / T_{0}\right)$ и $\eta=\eta_{0} \exp \left(-T / T_{1}\right)$ была определена характеристическая температура порогового тока $T_{0}=205 \mathrm{~K}$ и характеристическая температура дифференциальной эффективности $T_{1}=235 \mathrm{~K}$ в температурном диапазоне $20-50^{\circ} \mathrm{C}$. Насколько нам известно, это наибольшее значение $T_{0}$ среди опубликованных для любых лазеров на основе InP (за исключением лазеров на КТ, в которых используется модулированное легирование $p$-типа $[11,15])$. Дальнейший рост температуры приводит к ухудшению величины $T_{0}$.

Характеристическая температура порогового тока $T_{0}$ для различных длин представлена на рис. 3. Она остается высокой для всех лазерных диодов с длиной 2 мм и больше. В 2-миллиметровом лазере достигалась $T_{0}$ величиной $191 \mathrm{~K}$. С дальнейшим уменьшением длины диода $T_{0}$ спадает до $140 \mathrm{~K}$.

На рис. 3 также представлены данные о длинах волн лазерной генерации. Образцы с длинами 2-4 мм излучают на длинноволновом склоне перехода основного состояния на длине волны около 1.49 мкм (см. также вставку к рис. 3). Лазерная генерация в 1-миллиметровых приборах требует большего усиления и лазерная линия смещается в сторону коротких длин волн (1.45 мкм), что типично для массивов КТ с большим неоднородным уширением [25]. Скорее всего, именно этот эффект и приводит к росту порогового тока и уменьшению дифференциальной эффективности в коротких лазерах (см. рис. 1). Принимая во внимание величину внутренних потерь и длину резонатора, при которой возникает синее смещение длины волны, можно оценить насыщенное усиление равным $\sim 18 \mathrm{~cm}^{-1}$.

Как следует из синего смещения длины волны излучения в коротких приборах, генерация в них возникает за счет состояний КТ с меньшей энергией локализации, что усиливает выброс носителей заряда из активной области в волноводный слой. По-видимому, этот эффект ответствен за уменьшение $T_{0}$.

Опубликованные значения $T_{0}$ для 1.5 мкм InAs/InP KT лазеров показаны на рис. 4 в зависимости от ширины запрещенной зоны $E_{g}$ материала матрицы (волновода). Хотя данные немного разнятся (это может быть связано

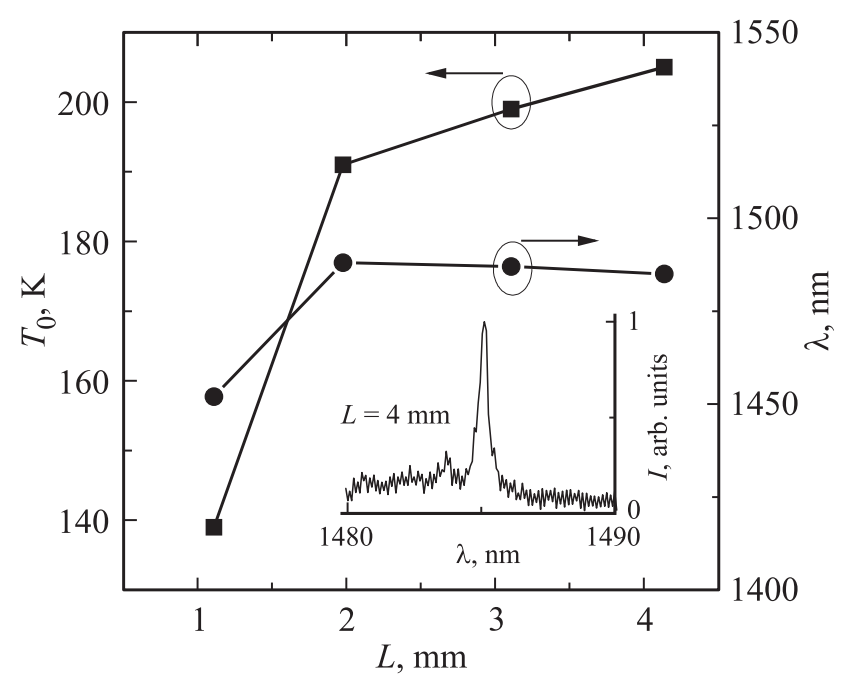

Рис. 3. Характеристическая температура $T_{0}$ и длина волны излучения в зависимости от длины резонатора. На вставке спектр лазерной генерации 4 мм лазера при $20^{\circ} \mathrm{C}$ и токе накачки, немного превышающем пороговый. 


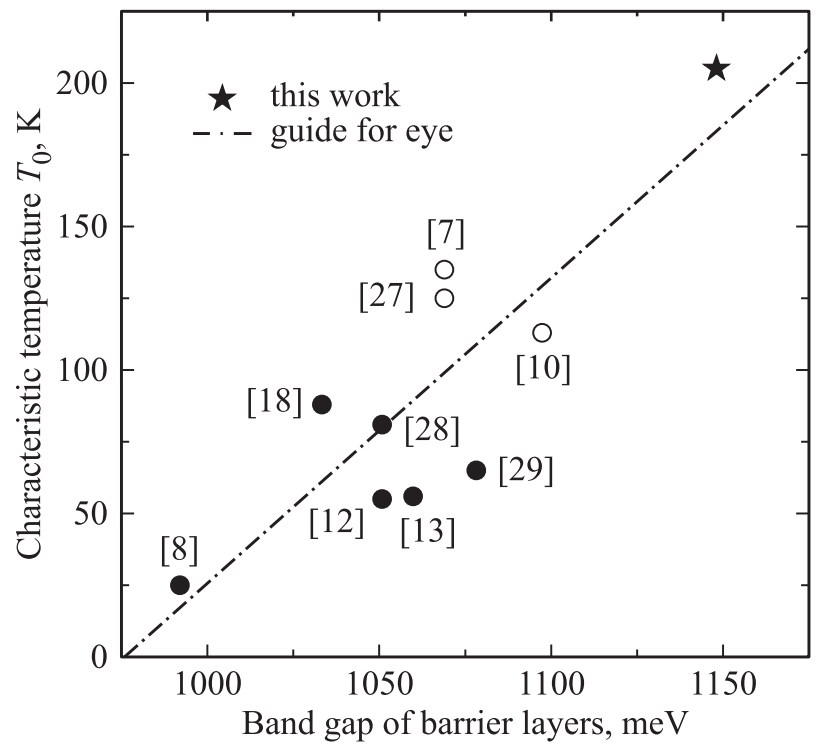

Рис. 4. Зависимость характеристической температуры $T_{0}$ от ширины запрещенной зоны волноводных слоев для 1.5 мкм InAs/InP КT лазеров (настоящая работа и работы $[7,8,10,12,13,18,27-29])$. Открытые символы соответствуют лазерам с InGaAlAs-волноводом, а сплошные символы InGaAsP.

с различием в геометрических размерах лазеров и других параметров), можно проследить общую тенденцию, которая заключается в росте $T_{0}$ с увеличением ширины запрещенной зоны (штриховая линия приведена для наглядности). Из этого следует, что заполнение лазерного волновода носителями заряда является одной из основных причин плохой температурной стабильности таких лазеров. В пользу этого вывода служит то обстоятельство, что наиболее значимым параметром с точки зрения паразитной рекомбинации в волноводе является разница между шириной запрещенной зоны $E_{g}$ и энергией лазерного оптического перехода $h v$, поскольку темп волноводной рекомбинации пропорционален $T^{3 / 2} \exp \left[\left(h v-E_{g}\right) /\left(k_{B} T\right)\right]$ [26]. Отметим, что на рис. 4 мы приводим данные, полученные для двух материалов лазерного волновода: InAlGaAs и InGaAsP, которые имеют различные разрывы в валентной зоне и зоне проводимости.

В большинстве работ, данные которых представлены на рис. 4, ширина запрещенной зоны волноводных слоев лежит в диапазоне $1.03-1.1$ эВ. Соответствующие значения $T_{0}$ попадают в интервал $55-135 \mathrm{~K}$. Наименьшая ширина запрещенной зоны $(0.99$ эВ) и наименьшая $T_{0}=25 \mathrm{~K}$ приведены в работе [8]. В настоящей работ была использована наибольшая ширина запрещенной зоны 1.15 эВ (что по меньшей мере на 50 мэВ больше, чем в остальных работах), что находится в согласии с наибольшей $T_{0}$ среди представленных.

Значение $T_{0}=205 \mathrm{~K}$, достигнутое в данной работе, сравнимо с характеристической температурой в InP КТ лазерах с модулированным p-легированием [11]
$\left(T_{0}=210 \mathrm{~K}\right)$ и $p$-легированием в сочетании с электронной туннельной инжекцией $[15]\left(T_{0}=227 \mathrm{~K}\right)$. Однако стоит отметить, что $p$-легирование приводит к дополнительным оптическим потерям за счет поглощения на свободных носителях, а лазерные структуры с туннельной инжекцией в активную область не получили широкого распространения вследствие трудности их реализации.

\section{4. Заключение}

Таким образом, нами продемонстрированы КТ лазеры на InP-подложке с гребешковым волноводом шириной 2 мкм с длиной волны излучения около 1.5 мкм. Была достигнута рекордно высокая характеристическая температура порогового тока $\left(T_{0}=205 \mathrm{~K}\right)$ среди лазеров на основе InP любого типа с нелегированной активной областью. Мы связываем этот результат с использованием в гетероструктуре лазера волновода/матрицы c большой энергией запрещенной зоны и ожидаем, что дальнейшее увеличение $T_{0}$ может быть достигнуто за счет использования асимметричных барьерных слоев [30]. Следует отметить, что высокая температурная стабильность была достигнута в лазерах с активной областью, обладающей большим неоднородным уширением, оптимизированной для использования в лазерах, работающих в режиме синхронизации мод.

Работа выполнена при поддержке РФФИ (грант № 16-29-03123). Е.С. Семенова и K.Yvind благодарны Villum Fonden (YIP QUEENs) и Европейской комиссии (FP7-IRSES HyMeCav).

\section{Список литературы}

[1] D. Bimberg, N. Kirstaedter, N.N. Ledentsov, Zh.I. Alferov, P.S. Kop'ev, V.M. Ustinov. IEEE J. Select. Topics. Quant. Electron., 3 (2), 196 (1997).

[2] V.M. Ustinov, N.A. Maleev, A.E. Zhukov, A.R. Kovsh, A.Yu. Egorov, A.V. Lunev, B.V. Volovik, I.L. Krestnikov, Yu.G. Musikhin, N.A. Bert, P.S. Kop'ev, Zh.I. Alferov, N.N. Ledentsov, D. Bimberg. Appl. Phys. Lett., 74 (19), 2815 (1999).

[3] M.G. Thompson, A. Rae, R.L. Sellin, C. Marinelli, R.V. Penty, I.H. White, A.R. Kovsh, S.S. Mikhrin, D.A. Livshits, I.L. Krestnikov. Appl. Phys. Lett., 88 (13), 133119 (2006).

[4] D.G. Deppe, K. Shavritranuruk, G. Ozgur, H. Chen, S. Freisem. Electron. Lett., 45 (1), 54 (2009).

[5] F.I. Zubov, M.V. Maximov, E.I. Moiseev, A.V. Savelyev, Y.M. Shernyakov, D.A. Livshits, N.V. Kryzhanovskaya, A.E. Zhukov. Electron. Lett., 51 (21), 1686 (2015).

[6] S. Fathpour, Z. Mi, P. Bhattacharya, A.R. Kovsh, S.S. Mikhrin, I.L. Krestnikov, A.V. Kozhukhov, N.N. Ledentsov. Appl. Phys. Lett., 85 (22), 5164 (2004).

[7] J.S. Kim, J.H. Lee, S.U. Hong, W.S. Han, H.-S. Kwack, C.W. Lee, D.K. Oh. IEEE Phot. Technol. Lett., 16 (7), 1607 (2004). 
[8] S. Anantathanasarn, R. Nötzel, P.J. van Veldhoven, F.W.M. van Otten, Y. Barbarin, G. Servanton, T. de Vries, E. Smalbrugge, E.J. Geluk, T.J. Eijkemans, E.A.J.M. Bente, Y.S. Oei, M.K. Smit, J.H. Wolter. Appl. Phys. Lett., 89 (7), 073115 (2006).

[9] A. Maryński, G. Sȩk, A. Musiał, J. Andrzejewski, J. Misiewicz, C. Gilfert, J.P. Reithmaier, A. Capua, O. Karni, D. Gready, G. Eisenstein, G. Atiya, W.D. Kaplan, S. Kölling. J. Appl. Phys., 114 (9), 094306 (2013).

[10] K. Akahane, N. Yamamoto, T. Kawanishi. IEEE Phot. Technol. Lett., 22 (2), 103 (2010).

[11] Y. Li, T.J. Rotter, Y.C. Xin, A. Stinz, A. Martinez, K.J. Malloy, S. Patterson, L.F. Lester. Conference on Lasers and ElectroOptics and 2006 Quantum Electronics and Laser Science Conf (Long Beach, CA, USA, 21-26 May 2006), CThX6.

[12] P. Caroff, C. Paranthoen, C. Platz, O. Dehaese, H. Folliot, N. Bertru, C. Labbé, R. Piron, E. Homeyer, A. Le Corre, S. Loualiche. Appl. Phys. Lett., 87 (24), 243107 (2005).

[13] F. Lelarge, B. Rousseau, B. Dagens, F. Poingt, F. Pommereau, A. Accard. IEEE Phot. Technol. Lett., 17 (7), 1369 (2005).

[14] C. Gilfert, V. Ivanov, N. Oehl, M. Yacob, J.P. Reithmaier. Appl. Phys. Lett., 98 (20), 201102 (2011).

[15] S. Bhowmick, M.Z. Baten, T. Frost, B.S. Ooi, P. Bhattacharya. IEEE J. Quant. Electron., 50 (1), 7 (2014).

[16] B. Jo, C.-R. Lee, J.S. Kim, W.S. Han, J.H. Song, J.-H. Choi, J.-H. Ryou, J.H. Lee, J.-Y. Leem. Laser Phys. Lett., 11 (11), 115815 (2014).

[17] Z.G. Lu, J.R. Liu, P.J. Poole, S. Raymond, P.J. Barrios, D. Poitras, G. Pakulski, P. Grant, D. Roy-Guay. Opt. Express, 17 (16), 13609 (2009).

[18] L. Shuai, J. Hai-Ming, G. Feng, Y. Xiao-Guang, L. Ping, Z. Ling-Juan, Y. Tao. Chin. Phys. Lett., 30 (6), 068101 (2013).

[19] K. Yvind, D. Larsson, J. Mørk, J.M. Hvam, M. Thompson, R. Penty, I. White. Proc. SPIE, 6909, 69090A (2008).

[20] F. Gao, S. Luo, H.-M. Ji, F. Xu, Z.-R. Lv, X.-G. Yang, P. Liang, T. Yang. Appl. Phys. Lett., 108 (20), 201107 (2016).

[21] A.R. Rae, M.G. Thompson, R.V. Penty, I.H. White, A.R. Kovsh, S.S. Mikhrin, D.A. Livshits, I.L. Krestnikov. 19th Annual Meeting Lasers and Electro-Optics Soc. (Montreal, Canada, 29 Oct. -2 Nov. 2006), ThR5.

[22] A.E. Zhukov, M.V. Maximov, A.V. Savelyev, Yu.M. Shernyakov, F.I. Zubov, V.V. Korenev, A. Martinez, A. Ramdane, J.-G. Provost, D.A. Livshits. J. Appl. Phys., 113 (23), 233103 (2013).

[23] M.Z.M. Khan, T.K. Ng, B.S. Ooi. Prog. Quant. Electron., 38 (6), 237 (2014).

[24] E.S. Semenova, I.V. Kulkova, S. Kadkhodazadeh, M. Schubert, K. Yvind. Appl. Phys. Lett., 99 (10), 101106 (2011).

[25] L.V. Asryan, M. Grundmann, N.N. Ledentsov, O. Stier, R.A. Suris, D. Bimberg. IEEE J. Quant. Electron., 37 (3), 418 (2001).

[26] L.V. Asryan, R.A. Suris. IEEE J. Quant. Electron., 34 (5), 841 (1998).

[27] J.P. Reithmaier, S. Banyoudeh, A. Abdollahinia, F. Schnabel, V. Sichkovskyi, O. Eyal, G. Eisenstein. Proc. SPIE, 9892, 98920A (2016).

[28] S.G. Li, Q. Gong, Y.F. Lao, K. He, J. Li, Y.G. Zhang, S.L. Feng, H.L. Wang. Appl. Phys. Lett., 93 (11), 111109 (2008).

[29] D. Franke, M. Moehrle, A. Sigmund, H. Kuenzel, U.W. Pohl, D. Bimberg. J. Appl. Phys., 109 (8), 083104 (2011).
[30] A.E. Zhukov, N.V. Kryzhanovskaya, F.I. Zubov, Y.M. Shernyakov, M.V. Maximov, E.S. Semenova, K. Yvind, L.V. Asryan. Appl. Phys. Lett., 100 (2), 021107 (2012).

Редактор Г.А. Оганесян

\section{High characteristic temperature of near-1 $\mu \mathrm{m}$ InAs/GaAs/InGaAsP quantum dot laser on InP substrate}

F.I. Zubov' ${ }^{1}$, E.S. Semenova ${ }^{2}$, I.V. Kulkova ${ }^{2}$, K. Yvind ${ }^{2}$, N.V. Kryzhanovskaya ${ }^{1}$, M.V. Maximov ${ }^{1}$, A.E. Zhukov ${ }^{1}$

${ }^{1}$ St. Petersburg Academic University,

194021 St. Petersburg, Russia

${ }^{2}$ Technical University of Denmark,

DTU Fotonics Department of Photonics Engineering,

DK-2800 Kgs. Lyngby, Denmark

Abstract We report on high temperature stability of a near$1.5 \mu \mathrm{m}$ laser synthesized on an $\operatorname{InP}(001)$ substrate. Self-organized InAs quantum dots capped with a thin GaAs layer were used as an active region of the laser. An InGaAsP quaternary alloy having the bandgap energy of $1.15 \mathrm{eV}$ was utilized as a waveguiding/matrix layer. A high characteristic temperature of the threshold current of $T_{0}=205 \mathrm{~K}$, evaluated in the temperature range of $20-50^{\circ} \mathrm{C}$, was achieved in ridge waveguide laser diodes. A correlation of $T_{0}$ values with the bandgap energy of the waveguiding layer was found. 